\title{
Identification and characterization of the GmRD26 soybean promoter in response to abiotic stresses: potential tool for biotechnological application
}

Elinea O. Freitas ${ }^{1,2+}$, Bruno P. Melo ${ }^{1,3 \dagger}{ }^{1}$, Isabela T. Lourenço-Tessutti ${ }^{1}$, Fabrício B. M. Arraes ${ }^{1,4}$, Regina M. Amorim', Maria E. Lisei-de-Sá ${ }^{1,5}$ D, Julia A. Costa ${ }^{1,6}$, Ana G. B. Leite ${ }^{1,2}$, Muhammad Faheem ${ }^{1,7}$, Márcio A. Ferreira ${ }^{8}$,

Carolina V. Morgante ${ }^{1,9}$, Elizabeth P. B. Fontes ${ }^{3}$ and Maria F. Grossi-de-Sa ${ }^{1,6^{*}}$

\begin{abstract}
Background: Drought is one of the most harmful abiotic stresses for plants, leading to reduced productivity of several economically important crops and, consequently, considerable losses in the agricultural sector. When plants are exposed to stressful conditions, such as drought and high salinity, they modulate the expression of genes that lead to developmental, biochemical, and physiological changes, which help to overcome the deleterious effects of adverse circumstances. Thus, the search for new specific gene promoter sequences has proved to be a powerful biotechnological strategy to control the expression of key genes involved in water deprivation or multiple stress responses.

Results: This study aimed to identify and characterize the GmRD26 promoter (pGmRD26), which is involved in the regulation of plant responses to drought stress. The expression profile of the GmRD26 gene was investigated by qRT-PCR under normal and stress conditions in Williams 82, BR16 and Embrapa48 soybean-cultivars. Our data confirm that GmRD26 is induced under water deficit with different induction folds between analyzed cultivars, which display different genetic background and physiological behaviour under drought. The characterization of the GmRD26 promoter was performed under simulated stress conditions with abscisic acid (ABA), polyethylene glycol (PEG) and drought (air dry) on A. thaliana plants containing the complete construct of pGmRD26::GUS (2.054 bp) and two promoter modules, pGmRD26A:GUS (909 pb) and pGmRD26B::GUS (435 bp), controlling the expression of the $\beta$-glucuronidase (uidA) gene. Analysis of GUS activity has demonstrated that $\mathrm{pGmRD26}$ and $\mathrm{pGmRD26A}$ induce strong reporter gene expression, as the pAtRD29 positive control promoter under ABA and PEG treatment.

Conclusions: The full-length promoter pGmRD26 and the pGmRD26A module provides an improved uidA transcription capacity when compared with the other promoter module, especially in response to polyethylene glycol and drought treatments. These data indicate that pGmRD26A may become a promising biotechnological asset with potential use in the development of modified drought-tolerant plants or other plants designed for stress responses.
\end{abstract}

Keywords: Stress-responsive promoter, Drought tolerance, Abscisic acid, Promoter modules analysis, Gene-promoter characterization

\footnotetext{
* Correspondence: mariafatimagrossidesa@gmail.com;

fatima.grossi@embrapa.br

${ }^{\dagger}$ Elinea O. Freitas and Bruno P. Melo contributed equally to this work.

${ }^{1}$ Embrapa Genetic Resources and Biotechnology, Brasília, DF, Brazil

${ }^{6}$ Catholic University of Brasilia - Post-Graduation Program in Genomic

Sciences and Biotechnology, Brasília, DF, Brazil

Full list of author information is available at the end of the article
}

(c) The Author(s). 2019 Open Access This article is distributed under the terms of the Creative Commons Attribution 4.0 International License (http://creativecommons.org/licenses/by/4.0/), which permits unrestricted use, distribution, and reproduction in any medium, provided you give appropriate credit to the original author(s) and the source, provide a link to the Creative Commons license, and indicate if changes were made. The Creative Commons Public Domain Dedication waiver (http://creativecommons.org/publicdomain/zero/1.0/) applies to the data made available in this article, unless otherwise stated. 


\section{Background}

Drought is one of the most limiting and severe abiotic stresses for field crops because it causes significant losses in plants production on a global scale $[1,2]$. Under drought conditions, plants trigger many physiological, biochemical, and molecular responses. The sign of abiotic stress is perceived by cellular receptors and secondary messengers, culminating in the gene expression reprogramming to improve plant tolerance, adaptation, and survival. In signal transduction cascade, transcription factors (TFs) emerge as one of the most important messengers in plant adaptation, because they are capable of modifying specific gene expression, encompassing different physiological changes [3, 4]. For example, abiotic factors such as drought, salinity, and heat (high evaporation) alter the osmotic balance in plants' cell, inducing the biosynthesis of abscisic acid (ABA), a vital phytohormone involved in the expression of droughtrelated genes $[5,6]$.

Several TFs are involved in water stress tolerance, including ABA-responsive element (ABRE), nitrogen assimilation control (NAC), dehydration-responsive element binding (DREB), basic leucine zipper (bZIP), myeloblastosis (MYB) and myelocytomatosis (MYC) proteins. All of these TFs are mediators of the classic ABA-dependent or ABAindependent signaling pathways [7-11]. These transcription factors bind preferentially to the dehydration-responsive element (DRE) core sequence (A/GCCGAC) of generesponsive promoters and regulate several stress-induced genes [12]. The DRE sequence is present in the $A$. thaliana $R D 29 A$ (AtRD29) promoter region and is used extensively to drive expression in a stress-inducible manner in different plants, such as tobacco [13], potato [14], and soybean [15].

The expression of DREB1A under the control of the $A t R D 29$ promoter in A. thaliana increased the survival rate of plants stressed with freezing, drought, high salinity, and high temperature [16]. Similar results were also observed in tobacco plants [13]. In both cases, the use of inducible promoter AtRD29 displays a higher gene expression than the Cauliflower mosaic virus 35S (CaMV35S) constitutive promoter, as it reduces the pleiotropic effects on growth due to the overexpression of DREB1A [13, 16]. The expression of genes of interest under the control of the AtRD29 promoter has been widely used to regulate drought toleranceassociated genes in different plant species [16-19]. However, compared with AtRD29, some specific inducible promoters can achieve higher levels of expression. An example is the promoter of the Coffea arabica CaHB12 gene [20]. For this reason, the identification, isolation, and characterization of new specific promoters inducible by abiotic stress have been crucial to ensure the successful application of gene modification and, consequently, the development of new cultivars resistant to water deprivation stress.

In this study, we have investigated the gene expression profile of GmRD26 in soybean (Glycine max), homologous to the A. thaliana AtRD26 gene (ANAC072). GmRD26 is highly induced by ABA, PEG, and drought. According to our gene expression analysis, it displays a similar expression profile in comparison with AtRD26 and GmNAC085, a soybean $G m R D 26$ gene-paralogue already characterized $[11,21]$. These genes belong to SNAC-A subfamily (ATAF), as well as GmRD29, the AtRD29 orthologue, extensively used as a model of drought-inducible genes. Previous studies of the GENOSOJA project have demonstrated that GmRD29 was not differentially expressed during severe water deprivation as GmRD26 [22], selected as the focus for this study.

Many $S N A C$ - $A$ genes are involved with different abiotic stress responses and senescence progression. In soybean, $44 \%$ of NAC genes are differentially expressed (DE) during age triggered senescence, being $90 \%$ of genes from SNACA subfamily [21]. In A. thaliana, all SNAC-A gene members - ANAC055 (AT3G15500), ANAC019 (AT1G52890), ANAC072/RD26 (AT4G27410), ANAC002/ATAF1 (AT1G 01720), ANAC081/ATAF2 (AT5G08790), ANAC102 (AT5 G63790), and ANAC032 (AT1G77450) - are induced by age triggered leaf senescence [21, 23]. AtRD26 acts as a transcriptional activator in ABA-mediated dehydration response, positively regulating NYE1, which triggers chlorophyll degradation [24]. The GmRD26 paralogue in soybean ( $G m N A C 085)$ is also a positive regulator of leaf senescence, displaying high expression during age triggered senescence and classic senescence symptoms when transiently expressed in Nicotiana benthamiana [21].

We subsequently isolated and characterized the GmRD26 promoter (pGmRD26). The transcriptional activity of pGmRD26 and its modules were evaluated in transgenic $A$. thaliana plants under the stress conditions with abscisic acid (ABA), polyethylene glycol (PEG) and drought (air dry) to evaluate the activities of the different regions of the pGmRD26.

\section{Results}

Soybean RD26 gene expression profile in distinct soybean lines under different stress conditions

To identify and characterize the orthologue gene of $A$. thaliana AtRD26 (ANAC072) in soybean, an in-silico approach was applied. The AtRD26 (AT4G27410) sequence was accessed and compared against the Williams 82 soybean reference genome. A putative RD26 orthologue (GmNAC043 - Glyma.06G248900) was identified by neighbour-joining analysis, which revealed that at least four genes (Glyma.13G279900, Glyma.12G221500, Glyma.06G248900, and Glyma.12G149100), closely related to AtRD26, are present in the soybean genome (Fig. 1). A comparative amino acid deduced sequence analysis of candidate genes was performed, and GmNAC043, called GmRD26, (Glyma.06G248900) displayed a relatively high amino acid similarity with $A t R D 26$. 


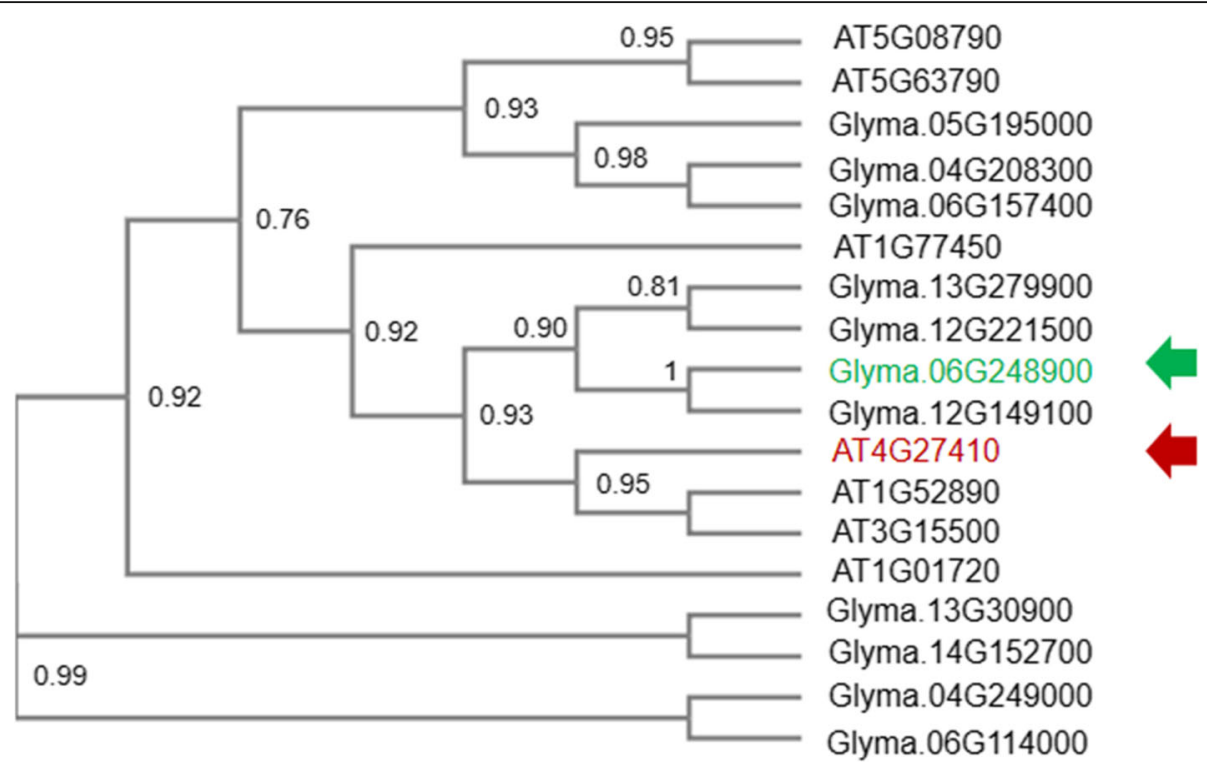

Fig. 1 Phylogenetic reconstruction of ATAF soybean genes, members of the NAC transcription factor subfamily. The deduced amino acid sequences of soybean and Arabidopsis were used to perform a multiple alignment using BLASTP and ClustalW2. The phylogenetic tree was constructed using MEGA4.0 software via the neighbour-joining method with a consensus of 10.000 bootstraps. The red arrow indicates the orthologue A. thaliana reference gene (AT4G27410), and the green arrow indicates GmRD26 (Glyma.06G248900)

To evaluate whether the GmRD26 soybean transcription factor is induced during water stress, its expression pattern was analyzed in cDNA subtractive libraries related to dissection experiments available in the GENOSOJA LGE (Genomics and Expression Laboratory: GENOSOJA Project) database and from these analyses, the presence of GmRD26 was confirmed. To evaluate the GmRD26 expression profile and its relation to multiple stress responses in soybean, the transcript levels were analyzed in the leaves and roots of Williams 82 soybean seedlings by qRT-PCR. The expression pattern was also evaluated for GmNAC085, a paralogue of GmRD26 gene whose stress induction profile is reported previously [21]. As expected, GmRD26 is highly expressed under the use of PEG $(10 \% \mathrm{~m} / \mathrm{v})$ in leaves but is also induced by ABA $(150 \mathrm{mM})$ and drought in leaves and roots (Fig. 2). This gene expression profile is similar to the GmNAC085 expression (Additional file 1: Figure S1). In addition, both related genes are repressed by tunicamycin (Tun) in leaves and roots, showing an expressive induction by salicylic acid (SA) $(5 \mathrm{mM})$ treatment in roots (Fig. 2; Additional file 1: Figure S1).

The expression profile of GmRD26 was also determined in two contrasting soybean genotypes in response to drought tolerance under simulated drought stress (Fig. 3) and ABA exogenous stimuli (Fig. 3a). It is expected that positive regulators of drought perception, signal transduction, and drought avoidance-associated genes are expressed higher in tolerant lineages than in susceptible lineages, as shown in the gene expression analysis results. In addition, the gene expression-folding is extensively high in BR16 and Embrapa48 cultivars when compared with the expression in Williams 82 .

GmRD26 was differentially expressed in both leaves and roots of contrasting cultivars, and the tissues display a similar induction pattern as observed in Williams 82 under PEG $(10 \% \mathrm{~m} / \mathrm{v})$ stress (Figs. 2 and 3). In the roots, the gene expression was considerably lower than in the leaves. The difference between the cultivars is the gene expression levels: the susceptible cultivar BR16 had a significantly lower GmRD26 transcript accumulation in comparison with the tolerant cultivar Embrapa48 at all times of stress progression (Fig. 3). The gene expression significantly increased beginning at $125 \mathrm{~min}$, showing that the GmRD26 gene is strongly induced under severe stress conditions. The ABA response was also analyzed. As observed in Arabidopsis, the results revealed that GmRD26 is also up-regulated by $\mathrm{ABA}$ in both soybean cultivars and the mRNA levels are significantly higher in tolerant cultivar Embrapa48, as observed in drought treatment (Fig. 3a and b).

\section{Analysis of water deficit-responsive cis-elements frequency}

To investigate the transcriptional activity of the GmRD26 soybean promoter under different stress conditions, the full-length promoter sequence $(2.054 \mathrm{bp})$ was analyzed using PLACE and Genomatix for cis-regulatory element mapping. The promoter sequence analysis revealed some conserved TATA- and CAAT-box regions that are essential for transcription initiation complex 

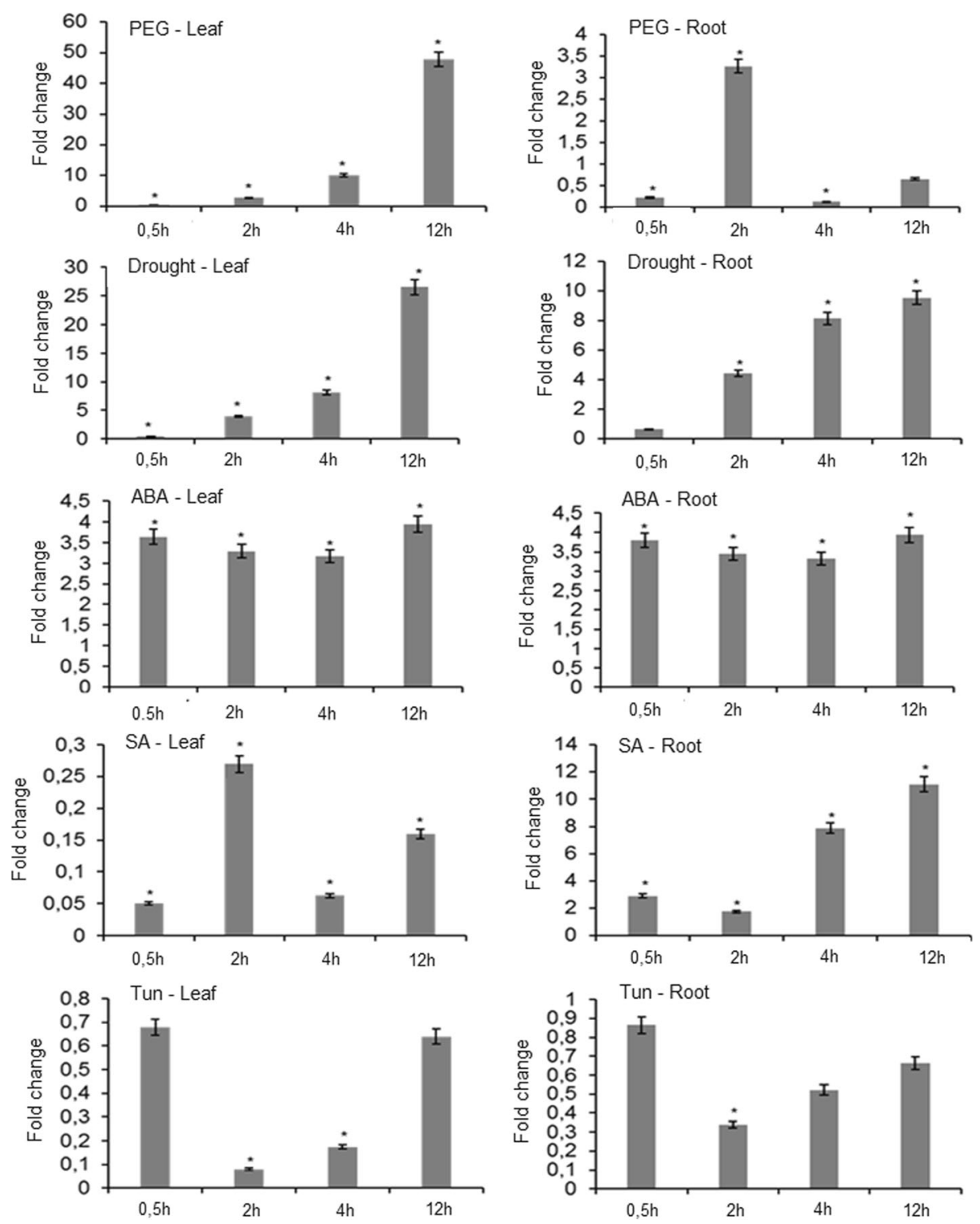

Fig. 2 GmRD26 expression profile in soybean (Williams 82) under multiple stresses. To determine the gene expression profile of the GmRD26 gene, the soybean seedlings were submitted to different stress conditions (ABA, PEG, SA, Tun and drought), and the gene expression in leaves and roots was analyzed by qRT-PCR. The fold change values were calculated in relation to untreated plants $(0 \mathrm{~h})$, considering the relative expression in these plants as 1. CYP2 and ELFTA were used as endogenous controls for normalization. The relative gene expression was calculated by the $2^{-\Delta \Delta C t}$ method in biological triplicates $(n=3)$. The bars represent standard errors and the asterisks $\left(^{*}\right)$ indicate statistical significance determined by the Student's $t$-test $(p \leq 0.05)$

assembly and gene transcription in eukaryotes. Potential cis-regulatory element families such as the ABRE, DREB, G-box, MYC and MYB families, which can respond to many environmental signals, abiotic stresses and phytohormones were also found in the pGmRD26 sequence (Fig. 4 and Table 1). The families' distribution in each promoter module used for $A$. thaliana genetic transformation is represented in Fig. 4b. Our analysis also revealed some specific drought-responsive cis-elements, MYB2AT and ACGTATERD1, as well as ABA-responsive ones, ABRERATCAL, ABREATCONSENSUS, DPBFCOREDCDC3, and EBOXBNNAPA Moreover, in the pGmRD26 sequences, some doubly responsive elements, MYB2CONSENSUSAT, ABREZMRAB28, MYBCORE, and G-box, have been identified that respond to both drought and ABA. (Fig. 5 and Table 1). The most frequent cis-elements identified in the modular pGmRD26A (909 bp) and pGmRD26B (435 bp) were DPBFCOREDCDC3, ABRERATCAL, and ABREATCONSENSUS, required in ABAsignaling and MYCCONSENSUSAT, ACGTATERD1 and MYBCORE, involved in dehydration-responses (Table 1). High-salinity responsive cis-elements are also present. 
(A)
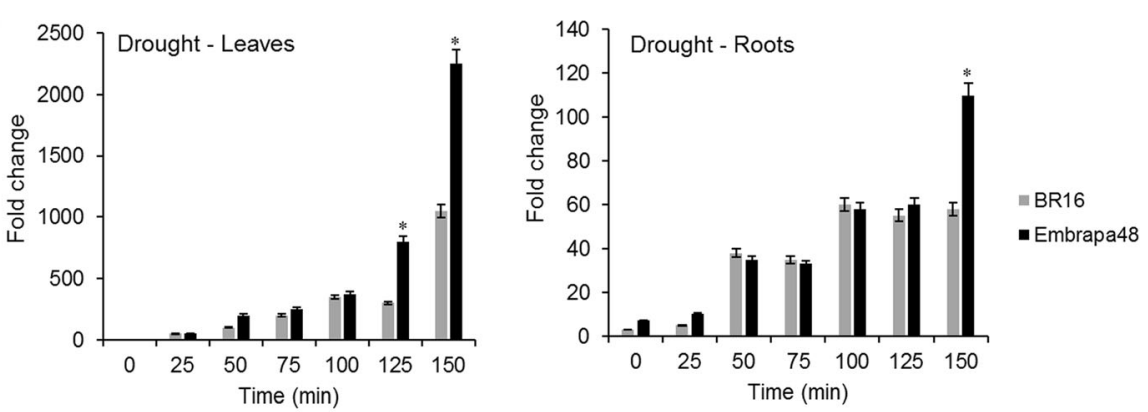

(B)

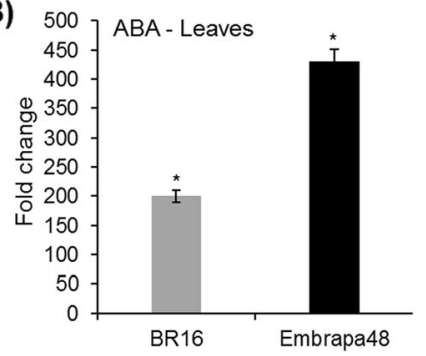

Fig. 3 GmRD26 expression profile in two soybean cultivars, the drought-susceptible BR16, and the drought-tolerant EMBRAPA48. a Expression profile of the GmRD26 gene under drought conditions, the gene expression pattern was determined at 25, 50, 75, 100, 125, and 150 min after water deprivation. $\mathbf{b}$ Expression profile of the GmRD26 soybean gene in the leaves of contrasting genotypes BR16 and EMBRAPA48 after $6 \mathrm{~h}$ of exogenous ABA stimuli. CYP2 and ELF1A were used as endogenous controls for normalization. The relative gene expression was calculated by the $2^{-\Delta \Delta C t}$ method in biological triplicates $(n=3)$. The bars indicate the standard errors and the asterisks $\left(^{*}\right)$ indicate statistical significance determined by Student's $t$-test $(p \leq 0.05)$

These stress-associated cis-elements were also found in AtRD29 promoter, and it was observed that GmRD26 promoter has nine of thirteen dehydration and ABAresponsive cis-elements, as found in $\mathrm{p} A t R D 29$ promoter (Additional file 2: Table S1).

\section{GUS activity and expression in transgenic $A$. thaliana} lineages under pGmRD26 control during different stress treatments

Homozygous $\mathrm{T}_{2} A$. thaliana lineages carrying the fulllength pGmRD26::GUS and the promoter modules pGmRD26A::GUS and pGmRD26B::GUS were used to analyse promoter induction under drought stress through GUS activity. The GUS histochemical assay was performed after $12 \mathrm{~h}$ of treatment with ABA and PEG in transgenic lineages and the controls $\mathrm{pAtRD} 29:$ :GUS (positive control) and non-treated plants (negative control). Plants carrying the pGmRD26::GUS and pGmRD26A:: GUS displayed intense GUS activity in their foliar vascular tissue after ABA treatment, as well the positive control pAtRD29 (Fig. 6A- a, e, m). In contrast, the pGmRD26B:: GUS is not strongly inducible by ABA, according to its GUS activity (Fig. 6A - i), although ABRE elements are abundantly distributed in this promoter module. Under

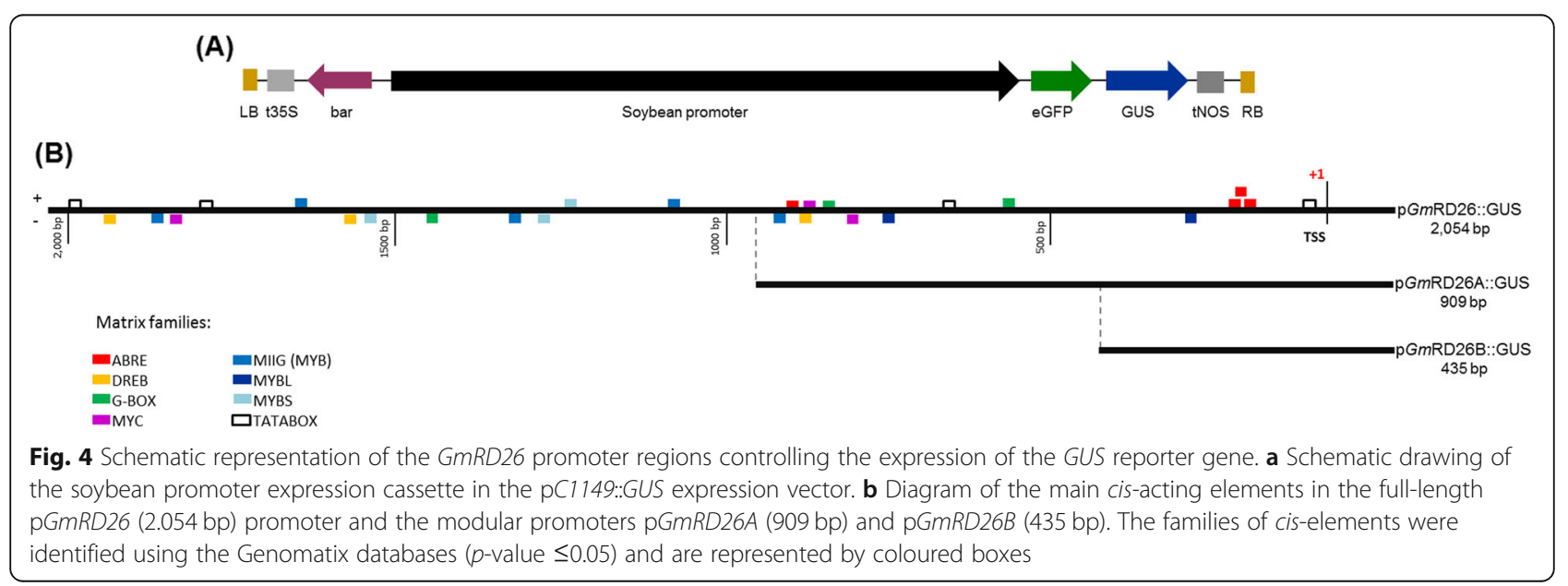


Table 1 Cis-regulatory elements related to drought in the pGmRD26 soybean promoter

\begin{tabular}{|c|c|c|c|}
\hline Cis-regulatory element & Core sequence & Description & References \\
\hline ACGTATERD1 & ACGT & Dehydration & [25] \\
\hline MYCCONSENSUSAT & CANNTG & Dehydration, ABA and Cold & {$[26,27]$} \\
\hline ACGTABREMOTIFA2OSEM & ACGTGKC & Dehydration and ABA & [28] \\
\hline DRE2COREZMRAB17 & ACCGAC & Dehydration and ABA & [29] \\
\hline MYB2CONSENSUSAT & YAACKG & Dehydration and ABA & [30] \\
\hline ABREZMRAB28 & CCACGTGG & ABA-responsive & [31] \\
\hline ABREATCONSENSUS & YACGTGGC & ABA-responsive & {$[32,33]$} \\
\hline MYBCORE & CNGTTR & Dehydration and ABA & {$[34,35]$} \\
\hline MYB1AT & WAACCA & Dehydration and ABA & [30] \\
\hline MYB2AT & TAACTG & Dehydration & [34] \\
\hline G-box & CACGTG & Dehydration, high salinity, ABA & [36] \\
\hline EBOXBNNAPA & CANNTG & ABA-responsive & {$[26,37]$} \\
\hline DPBFCOREDCDC3 & ACACNNG & ABA-responsive & {$[38,39]$} \\
\hline ABRERATCAL & MACGYGB & ABA-responsive & [40] \\
\hline
\end{tabular}

$\mathrm{K}=\mathrm{G} / \mathrm{T} ; \mathrm{R}=\mathrm{G} / \mathrm{A} ; \mathrm{W}=\mathrm{A} / \mathrm{T} ; \mathrm{N}=\mathrm{A} / \mathrm{C} / \mathrm{G} / \mathrm{T} ; \mathrm{Y}=T \mathrm{C}$

PEG treatment, the GUS activity pattern was similar (Fig. 6A - $\mathrm{b}, \mathrm{f}, \mathrm{j}, \mathrm{n}$ ), with a discrete decline in activity in the pGmRD26::GUS plants when compared with ABA and drought (air dry) treatment (Fig. 6A - b). In the pGmRD26A::GUS construct, a strong GUS-derived staining was observed in almost all the leaves surfaces in PEG treatment (Fig. 6A - f). Under the drought treatment, GUS activity was strongly detected in all analyzed leaves, mainly in the modular constructs pGmRD26A::GUS, pGmRD26B::GUS (Fig. 6A - g and k). The basal expression in the control plants (without stress conditions) was low but detectable (Fig. 6A $\mathrm{d}, \mathrm{h}, \mathrm{l}$, and $\mathrm{p}$ ). In our study, $p$ GmRD26A displays activity in all treatments, but this activity is higher under desiccation conditions (Fig. 6A - g), reinforcing the role of RD26 in desiccation-triggered protective mechanisms in plants.

To confirm the induction profile of pGmRD26 revealed by histochemical assays, GUS activity was also monitored in transgenic lineage plants. Under ABA treatment, fulllength pGmRD26 encompassed the same results when compared to the pAtRD29 positive control, and the module pGmRD26A displays the higher GUS activity (Fig. 6B). These results contrast with the PEG treatment, in which the full-length promoter and the pGmRD26A module exhibit higher activity when compared with the positive control, pAtRD29, and the pGmRD26B (Fig. 6B). When GUS activity was analyzed under drought treatment, pGmRD26A shows the same activity of the full-length promoter, higher than the smaller module pGmRD26B, but lower than the positive control. The activity of the pGmRD26A module was higher than the other fragments, and the pAtRD29 control under ABA and PEG treatments (Fig. 6B). In addition, pGmRD26A transgenic lines display high levels of uidA mRNA after PEG treatment, while pGmRD26 lines display high levels of uidA transcripts under ABA treatment. In the drought treatment, $\mathrm{p} A t R D 29$ control lines presented higher expression level than the pGmRD26 promoter and its modules. However, when we analyzed the differences between the three fragments after drought treatment, pGmRD26A showed higher expression levels compared to pGmRD26 and pGmRD26B (Fig. 7).

The results of qPCR demonstrate that transcriptional GUS activity, driven by the promoters pGmRD26 and pGmRD26A was similar but not the same during ABA treatment. According to our data, pGmRD26 display a similar expression when compared with pAtRD29 (positive control) and higher mRNA accumulation when compared with pGmRD26A module (Fig. 7a). This data is compatible with the histochemical assay. Under PEG treatment, the module pGmRD26A displayed a higher transcriptional activity, followed by the full-length pGmRD26 and the positive control pAtRD29 (Fig. 7b). The module pGmRD26B continued to displaying lower GUS transcriptional activity. As expected, under drought condition, pAtRD29 displayed higher GUS expression, followed by considerable GUS expression driven by modules pGmRD26A and pGmRD26B and the fulllength promoter pGmRD26 (Fig. 7c).

\section{Discussion}

In this study, we confirmed that the GmRD26 gene is induced under different simulated drought conditions. In view of the potential of this gene as a target for the development of strategies for the genetic engineering of resistant plants, we decided to isolate and characterize the GmRD26 promoter region. Our results show that pGmRD26 and its modules activated the reporter gene uidA under different water deprivation stress conditions. These results are consistent with the characteristics of 


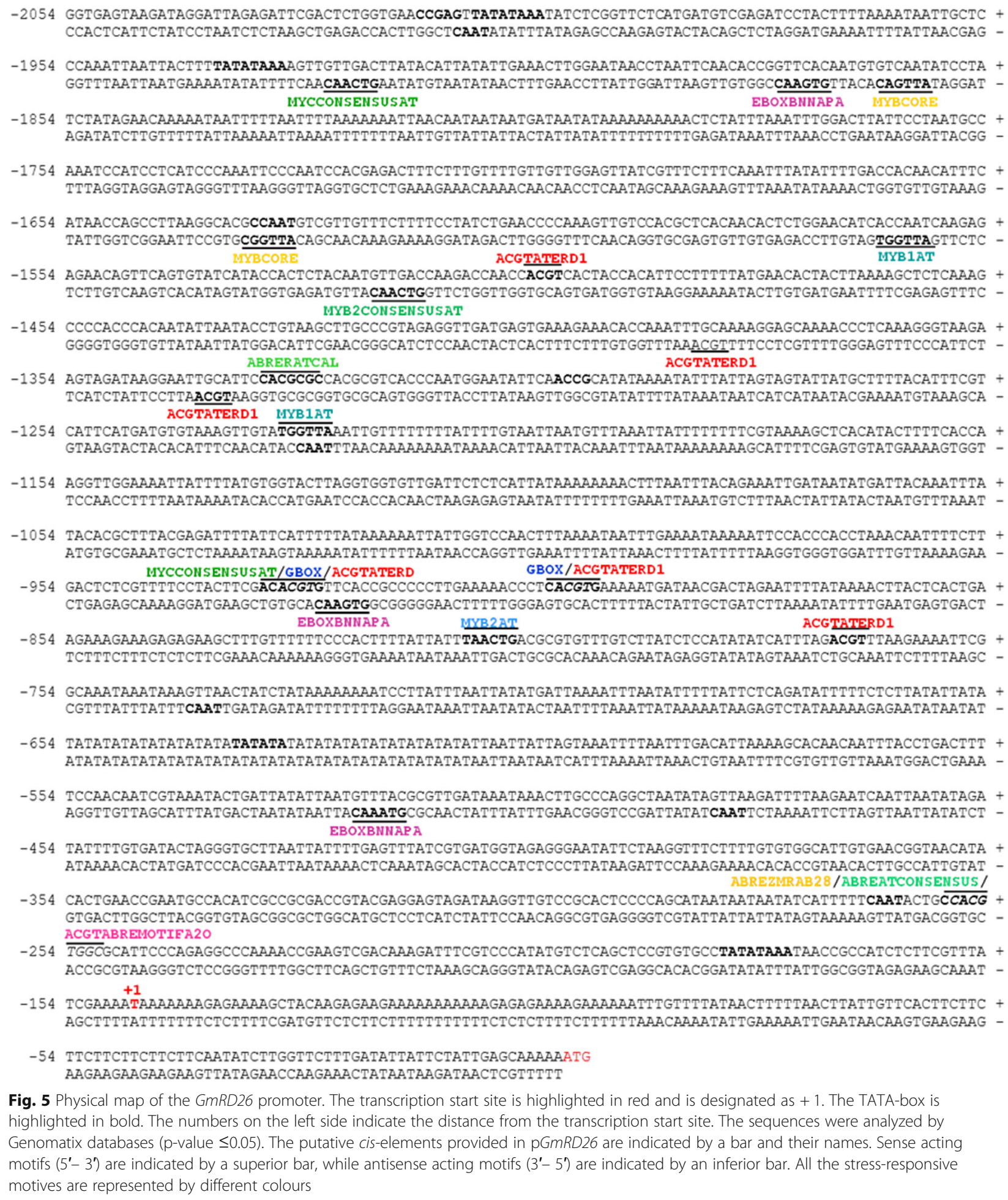

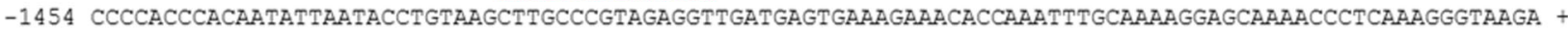
GGGGTGGGTGTTATA_ATTATGGACATTCGA_ACGGGCATCTCCA.ACTACTCACTTTCTTTGTGGTTTA_A_ACGTTTCCTCGTTTTGGGAGTTCCCATTCT ABRERATCAL $\quad$ ACGTATERDI

-1354 A.GTAGATAAGGA.ATTGCATTCCACGCGCCACGCGTCACCCA.ATGGA.ATATTCAACCGCATATAAAATATTTATTAGTAGTATTATGCTTTTACATTTCGT + TCATCTATTCCTTAACGTA.AGGTGCGCGGTGCGCAGTGGGTTACCTTATAAGTTGGCGTATATTTTA.TA.A.TA.TCA.TCA.TA.ATACGA.AA.TGTA.A.GCA. ACGTRTERD1 MYB1AT

-1254 CATTCATGATGTGTAAAGTTGTAFGGTTAAATTGTTTTTTTTATTTTGTAATTAATGTTTAAATTATTTTTTTTCGTAAAAGCTCACATACTTTTCACCA +

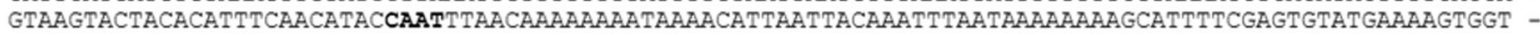

-1154 AGGTTGGAAATTATTTTATGTGGTACTTAGGTGGTGTTGATTCTCTCATTATAAAAAAAACTTTAATTTACAGAAATTGATAATATGATTACAAATTTA +

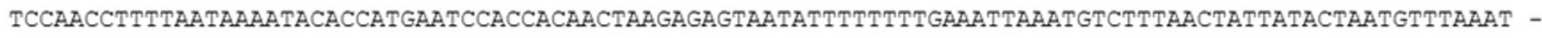

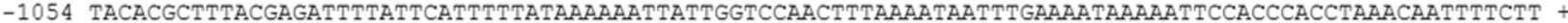

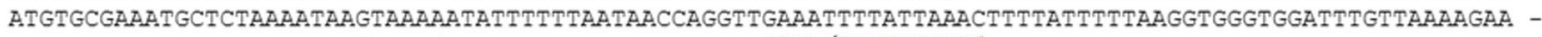
MYCCONSENSUSAT/GBOX/ACGTATERD GBOX/ACGTATERDI

-954 GACTCTCGTTTTCCTACTTCGACACGTGTTCACCGCCCCCTTGAAAAACCCT CACGTGAAAAATGATAACGACTAGAATTTTATAAAACTTACTCACTGA + CTGA.GA.GCA.A.A.GGATGA.ACTTGTGCA.CAAGTGGCGGGGGA.ACTTTTTGGGA.GTGCACTTTTTA.CTA.TTGCTGATCTTA.A.A.AATTTTGA.ATGA.GTGACT EBOXBNNAPA MXX2AT ACGTATERD1

-854 AGAAAGAAGAGAGAAGCTTTGTTTTTCCCACTTTTATTATTTAACTGACGCGTGTTTGTCTTATCTCCATATATCATTTAGACGTTTAAGAAAATTCG +

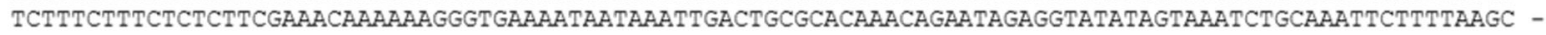

-754 GCAAATAAATAAAGTTACTATCTATAAAAAAAATCCTTATTTAATTATATGATTAAAATTTAATATTTTTATTCTCAGATATTTTTCTCTTATATTATA +

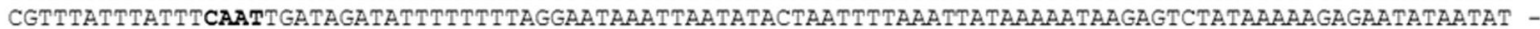

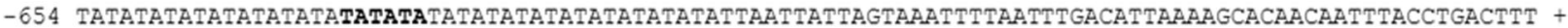

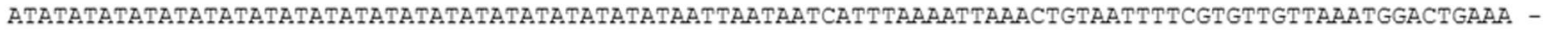

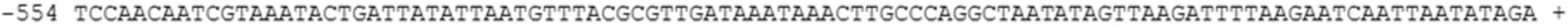
AGGTTGTTAGCATTTATGACTA.TATA.TTA.CAAATGCGCA.ACTATTTATTTGA.ACGGTCCGATTATATCAATTCTA.A.ATTCTTAGTTA.ATTATATCT EBOXBNNAPA

-454 TATTTTGTGATACTAGGTGCTTAATTATTTTGAGTTTATCGTGATGGTAGAGGAATATTCTAAGGTTTCTTTTGTGTGGCATTGTGAACGGTAACATA -

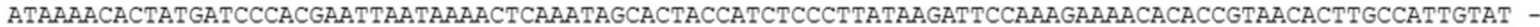
ABREZMRAB28/ABREATCONSENSUS/

-354 CACTGAACCGAATGCCACATCGCCGCGACCGTACGAGGAGTAGATAAGGTTGTCCGCACTCCCCAGCATAATAATAATATCATTTTTCAATACTGCCACG + GTGACTTGGCTTACGGTGTAGCGGCGCTGGCATGCTCCTCATCTATTCCAACAGGCGTGAGGGGTCGTATTATTATTATAGTAAAAAGTTATGACGGTGC ACGTABREMOTIFA2O

-254 TGGCGCATTCCCAGAGGCCCA.AA_CCGA.AGTCGACAAAGATTTCGTCCCATATGTCTCAGCTCCGTGTGCCTATATAAATA.ACGCCATCTCTTCGTTTA + ACCGCGTAAGGGTCTCCGGGTTTTGGCTTCAGCTGTTTCTA_AAGCAGGGTATACAGAGTCGAGGCACACGGATATATTTATTGGCGGTAGA.GA.AGCAAAT -

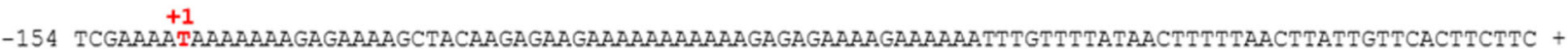

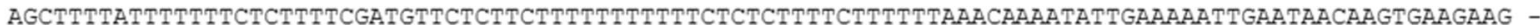

-54 TTCTTCTTCTTCTTCA.ATATCTTGGTTCTTGATATTATTCTATTGAgCA.A.A.A.TG AA.GA.GA.GAAGA.GTTATAGA.CCA.GAAACTATAATA.A.GAA.CTCGTTTTT

Fig. 5 Physical map of the GmRD26 promoter. The transcription start site is highlighted in red and is designated as + 1. The TATA-box is highlighted in bold. The numbers on the left side indicate the distance from the transcription start site. The sequences were analyzed by Genomatix databases ( $p$-value $\leq 0.05$ ). The putative cis-elements provided in pGmRD26 are indicated by a bar and their names. Sense acting motifs $\left(5^{\prime}-3^{\prime}\right)$ are indicated by a superior bar, while antisense acting motifs $\left(3^{\prime}-5^{\prime}\right)$ are indicated by an inferior bar. All the stress-responsive motives are represented by different colours

the cis-regulatory elements identified by in silico analyses of pGmRD26 sequence. In Arabidopsis, AtRD26 is an important member of ABA-dependent drought tolerance, and its overexpression is associated with a drought-tolerant phenotype [41].
The GmRD26 gene, as well as its A. thaliana orthologue (AtRD26/ANAC072), belongs to the subfamily SNAC-A, whose members have some correlation of functional conservation with the ATAF1 gene (AT1G01720), which has been shown to be a regulator of ABA biosynthesis and 


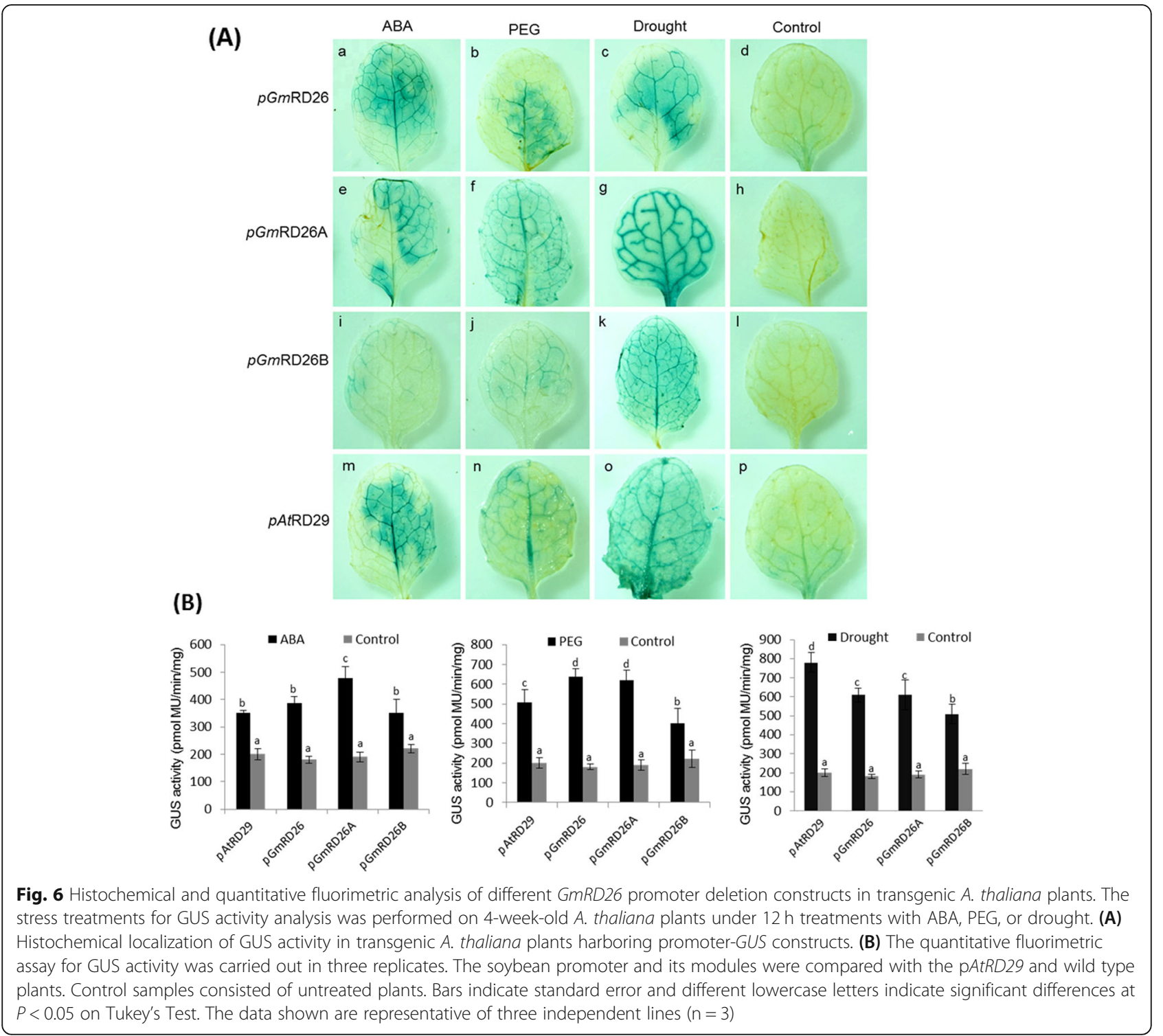
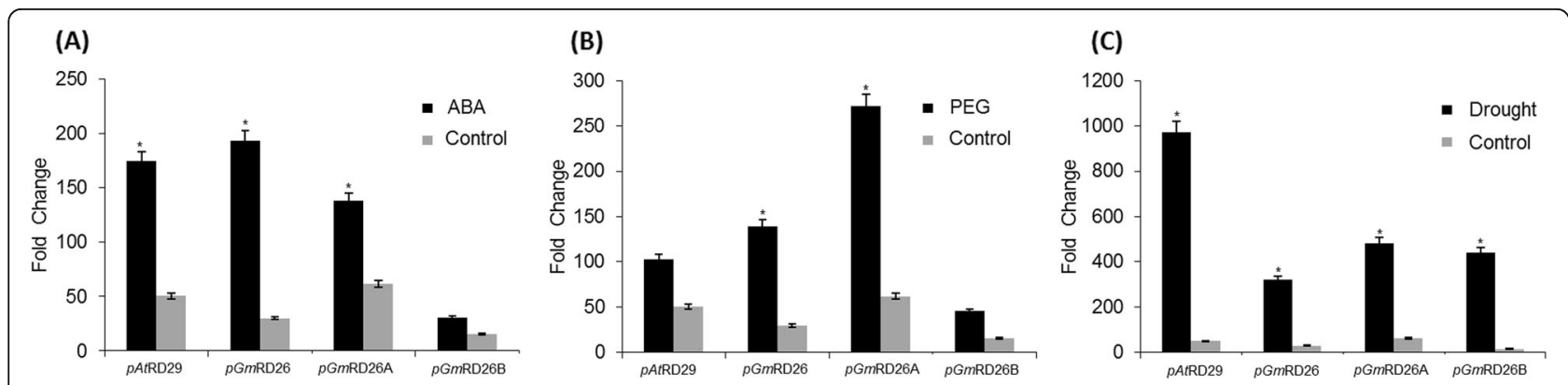

Fig. 7 Transcriptional GUS activity in transgenic A. thaliana under $12 \mathrm{~h}$ of ABA (a), PEG (b) or drought (c) treatments. Levels of uidA mRNA of nontreated (control) and treated transgenic plants were measured using real-time PCR. The expression levels were normalized using ACT2 and GAPDH as endogenous controls. The relative gene expression was calculated using the $2^{-\Delta \Delta C t}$ method. The bars represent standard error and the asterisks $\left(^{*}\right)$ indicate statistical significance determined by Student's $t$-test $(p \leq 0.05)$. The data shown are representative of three independent lines $(n=3)$ 
responsive to water stress [42-46]. Analysis of GmRD26 expression in two contrasting drought-tolerant soybean cultivars, BR16 and Embrapa48, and in the Williams 82 (soybean reference genome) demonstrated that the expression profile of the $G m R D 26$ gene is compatible with phylogenetic and molecular characteristics already described for orthologue genes, reinforced by GmNAC085 expression profile, a phylogenetically close-related gene in soybean [42, 47]. Our gene expression analysis reveals that the induction fold of GmRD26 is not the same along the three analyzed cultivars, displaying an unexpectedly high expression level in BR16 and Embrapa48. The different genetic background of these cultivars should proportion this difference, once the BR16 and Embrapa48 are commercial cultivars, obtained by genetic breeding programs. In our study, GmRD26 was responsive to osmotic stress during PEG treatment, desiccation and exogenous ABA stimulation in both leaves and roots, while the tolerant soybean variety displays higher gene expression level than susceptible variety. The leaves exhibited a more significant folding variation, suggesting that the physiological mechanism triggered by GmRD26, mainly related to ABAdependent responses, is more effective in the leaves than in the roots. In general, genes involved in ABA-mediated stress responses are involved in leaf morphophysiological changes, including stomatal closure, leaf area adjustment, photosynthesis, transpiration index, and osmolyte accumulation [48, 49]. In Arabidopsis, it was already demonstrated that GmNAC085 overexpression confers drought tolerance, improving the plant physiological performance during water deprivation stress. The transgenic lineages display a more robust antioxidative response under stress and many readouts genes, involved in ABA-dependent signalling, are up-regulated [11]. These results, associated with the determined GmRD26 and GmNAC085 gene expression profile, may justify the drought inducibility of SNAC-A genes during abiotic stress and confirm their potential to drive expression of genes involved with plant adaptability.

Compared with $\mathrm{p} A t R D 29$, a previously characterized drought-associated promoter, the promoter pGmRD26 was also enriched in stress-related cis-elements, responsive to salinity, dehydration, ABA and temperature. These results can be directly related to the excellent performance of the soybean promoter under ABA and PEG treatments. During drought treatment, the promoter displays some reasonable activity but is not capable of being compared with $\mathrm{p} A t R D 29$. This broad responsive promoter activity can be applied in soybean molecular breeding programs. In ABA-dependent pathways, ABREs (ABA-responsive elements) are the main phytohormone-responsive cis-element [43]. The occurrence of three ABRE motifs from five total ABA-responsive elements indicates a strong promoter induction under drought conditions, which can trigger increased drought-responsive gene expression by
pGmRD26 during stress. This effect is reinforced by the presence and frequency of the ACGT motif, a characteristic and important cis-element in drought-responsive promoters [50]. Cis-acting elements of the G-box family, found in several plant genes' promoters are known to interact with bZIP transcription factors, mediating responses to different stimuli. Studies comparing the patterns and evolution of the G-box family core (ACGT) in $O$. sativa, S. bicolor, A. thaliana and G. max suggest that this is the family with the most conserved elements between species and leads to responses to exogenous stresses, especially water and salt stress [51]. Other stressresponsive elements are also present in pGmRD26, such as MYCs/MYBs, which exhibit rapid induction in response to $\mathrm{ABA}$ treatment and water stress. These elements are targets of a large TF family in soya. MYC and MYB transcription factors are necessary for the early response to osmotic stress $[43,52]$.

In this study, we also the activity of the soybean promoter pGmRD26 and two-promoter modules, pGmRD26A and pGmRD26B, in transgenic A. thaliana plants that were submitted to simulated (ABA and PEG) and real drought stress. The pGmRD26, pGmRD26A, and pGmRD26B promoters were induced by all stress treatment assays, showing greater or similar GUS activity than pAtRD29 (positive control) under ABA, PEG, and drought treatment. Differences in induction intensity between pGmRD26 and modules under different types of abiotic stress are probably related to the distribution of specific cis-elements in their sequences involved in the control of water stress response [53, 54]. The transcriptional activation of some genes depends not only on the promoters' cis-acting elements and their sequences but also on their position and the presence of enhancers, regulatory sequences and other synergistic cis-elements $[55,56]$. It is important to highlight that some differences between transcriptional and translational activity are common on promoter's genes analysis. Our data demonstrate that the induction profile of pGmRD26 is similar in the tested conditions, showing that the fulllength promoter and its modules respond to the same conditions. Also, promoters' modules enriched in cisacting elements drive more consistent gene expression, reinforcing the idea of a synergistic effect of cis-elements in gene promoter sequences.

Similar results were obtained in the characterization of the $\alpha$-galactosidase soybean promoter (GlymaGAL) responsive to water stress; the smallest fragment, pGAL-1 $\mathrm{kb}$, showed no significant difference in GUS activity compared to the control and treated samples (PEG and dry). The full-length fragment promoter, $\mathrm{p} G A L-2 \mathrm{~kb}$, however, led to a significant increase in GUS expression. This increase in GUS expression of pGAL-2 kb was associated with a high number of ABRE, MYCATERD1, G- 
box, and DRE cis-elements [57]. Other studies have also reported the importance of distal promoter regions in responses associated with water stress in other species $[58,59]$.

\section{Conclusions}

In this study, we analyzed the expression profile of GmRD26 gene, which is expressed under simulated osmotic and drought conditions in soybean. The stressed soybean seedlings display a high GmRD26 expression under ABA exogenous-stimuli in leaves and roots, as well under PEG and air dry treatment. This gene expression pattern raised the hypothesis of drought-inducible cis-elements enriched promoter of GmRD26. Our analysis showed that the GmRD26 promoter region is enriched with essential cis-elements associated with drought stress, such as ABRE, DREB, MYB, MYC, and G-BOX. Molecular characterization of pGmRD26 in $A$. thaliana has demonstrated that the full promoter (pGmRD26) and two different promoter-modules (pGmRD26A and pGmRD26B) are inducible under simulated osmotic and drought stress conditions, confirming the soybean gene expression profile. In addition, our data also revealed that the full-length promoter and the pGmRD26A module, with higher cis-acting elements incidence compared to the other module, displayed a slightly higher level of expression than pGmRD26B and the $\mathrm{pAtRD29}$, an $A$. thaliana promoter used as a model to drought inducible gene studies, during ABA and PEG treatment. The complete characterization of pGmRD26 and its modules suggests that the promoter or the fragment pGmRD26A may become a potential biotechnological tool capable of inducing expression of genes of interest under specific conditions, such as drought or other abiotic stresses related with an osmotic imbalance to improve the tolerance associated to physiological performance in genetically modified plants.

\section{Methods \\ Identification of the drought marker gene GmRD26 in soybean}

The A. thaliana RD26 (AT4G27410) deduced amino acid sequence (available in TAIR database - https://www.arabidopsis.org/) was used to identify the closely related orthologue gene (GmRD26/GmNAC043 - Glyma.06G248900) in the soybean genome (Williams.82 v2.2-available in Phytozome: https://phytozome.jgi.doe.gov) [60]. For sequence comparison, BLASTP (https://blast.ncbi.nlm.nih.gov) was used, and the alignment was confirmed using the online tool ClustalW2 (https://www.ebi.ac.uk/Tools/msa/clustalw2). To determine the phylogenetic relationship between the Arabidopsis and soybean genes, the neighbourjoining clustering method derived from a distance matrix from a Poisson model was used, and the tree was reconstructed using MEGA software [61].

To evaluate whether the selected putative soybean gene GmRD26 is induced during drought stress, its expression pattern was analyzed from cDNA subtractive libraries related to dissection experiments available in the GENOSOJA database LGE (Genomics and Expression Laboratory: GENOSOJA Project) [22].

\section{Soybean plant growth conditions and stress treatments}

For the GmRD26 gene expression profile analysis, soybean (Williams 82) seeds were germinated in the soil and grown under greenhouse conditions (12 h of light, $25-35^{\circ} \mathrm{C}, 70 \%$ relative humidity) until the V2-V3 development stage. To simulate multiple stress conditions, the seedlings were first transferred to Hoagland hydroponic solution for $24 \mathrm{~h}$. After acclimation, the soybean roots were immersed in the same solution supplemented with $10 \%(\mathrm{w} / \mathrm{v})$ PEG 8000 to induce osmotic stress, $5 \mu \mathrm{g} /$ $\mathrm{mL}$ tunicamycin (Tun) to induce endoplasmic reticulum stress, $150 \mathrm{mM} \mathrm{ABA}$ and $5 \mathrm{mM}$ salicylic acid (SA) to simulate drought and biotic stress conditions, respectively. For the drought treatment, the plants were removed from the hydroponic solution and placed on plates with cotton. Leaf discs and roots of treated and control ( $0 \mathrm{~h}$ - untreated plants were collected one time) seedlings were collected after $0.5 \mathrm{~h}, 2 \mathrm{~h}, 4 \mathrm{~h}$ and $12 \mathrm{~h}$ of stress treatment and immediately frozen in liquid nitrogen. All treatments were performed at a three-plants pool, and samples were collected in triplicate.

Embrapa48 and BR16 soybean cultivars were used to determine the expression profile during the $\mathrm{ABA}$ and drought treatments in drought responses contrasting soybean lineages. The BR16 variety is considered as a model of drought sensitivity, while Embrapa48 is considered as drought-tolerant cultivar. The seeds were germinated in watered germination test paper and then transferred to a hydroponic box system filled with Hoagland solution. The seedlings in stages V3-V4 were grown under the same conditions as the Williams 82 seedlings. The drought stress was generated by removing the plants from the hydroponic solution and placing them in empty boxes for different water deprivation periods: 0 min (T0 - control), $25 \mathrm{~min}$ (T25), $50 \mathrm{~min}$ (T50), $75 \mathrm{~min}$ (T75), $100 \mathrm{~min}$ (T100), $125 \mathrm{~min} \mathrm{(T125)} \mathrm{and} 150 \mathrm{~min}$ (T150). Roots and leaf disc samples from three plants of each cultivar were collected during the exposure to water and were immediately frozen in liquid nitrogen for RNA extraction and gene expression analysis.

The contrasting soybean cultivars were also submitted to exogenous ABA treatment. Plants germinated and grown under the same conditions were sprayed with water (control) or ABA solution $(300 \mathrm{ppm})$. Three biological replicates were used, consisting of three plants 
per treatment. After $6 \mathrm{~h}$, leaf discs were collected and immediately frozen in liquid nitrogen for RNA extraction.

\section{RNA extraction, cDNA synthesis and GmRD26 gene expression analysis}

The total RNA of soybean leaf and roots was extracted according to the $\mathrm{TRIzol}^{\odot}$ manual (Invitrogen, USA). RNA quantification was performed using a NanoDrop ${ }^{\text {тм }}$ Spectrophotometer ND-1000 (Thermo Scientific, USA) and the RNA integrity was assessed by $1 \%$ agarose gel electrophoresis. A total of $2 \mu \mathrm{g}$ of RNA was used for cDNA synthesis with MMLV reverse transcriptase protocol (Invitrogen, USA).

The gene expression profile was determined by qPCR. The analysis was performed using an ABI 7500 Fast instrument, SYBR Green (Invitrogen, USA) reagent, specific primers (Additional file 3: Table S2) and three independent cDNA pools. All the analyses were performed using three biological and two technical replicates, originated from a five soybean plants pool. The reaction was performed as follow: $2 \mathrm{~min}$ at $50{ }^{\circ} \mathrm{C}, 10 \mathrm{~min}$ at $95^{\circ} \mathrm{C}$, and 40 cycles of $94{ }^{\circ} \mathrm{C}$ for $15 \mathrm{~s}$ and $60^{\circ} \mathrm{C}$ for 1 min. The CYP and ELF soybean genes [62] were used as endogenous controls for expression normalization and relative gene expression calculated by the $2^{-\Delta \Delta C t}$ method. The endogenous gene stability was determined by Gnorm algorithm (https://genorm.cmgg.be/), from Q-base package, and the M-value is 0.89 and 0.91 for $C Y P$ and $E L F$, respectively. The GmRD26 orthologue gene, GmNAC085, was used as a comparative control in Williams 82 for gene profile determination.

\section{Analysis of pGmRD26 soybean cis-acting elements} The pGmRD26 promoter sequence $(2.054 \mathrm{bp})$ was obtained from the soybean genome available in the Phytozome database (https://phytozome.jgi.doe.gov) [60]. The cis-acting elements responsive to drought-, salinity-, osmotic- and ABA-induced stress were identified, analyzed, and mapped using the Genomatix (https://www. genomatix.de/online_help/matinspector/matinspector). For this study, we considered only the cis-elements statistically significant, with a $p$-value $\leq 0.05[63,64]$.

\section{Construction of pGmRD26 plasmids}

The full-length GmRD26 soybean promoter region was considered as the $2.054 \mathrm{bp}$ gene-promoter, and A and B promoter-modules contain $909 \mathrm{bp}$ and $435 \mathrm{bp}$, respectively, considering the distribution of the droughtresponsive cis-acting elements. The sequences were transcriptionally fused in frame to the GUS gene in a binary expression pC1407 vector backbone, synthesized by Epoch Biolabs (Sugar Land, TX, USA). The generated recombinant plasmids were called pGmRD26::GUS (2.054 bp),
pGmRD26A::GUS (909bp) and pGmRD26B::GUS (435 $\mathrm{bp})$. The plasmids carry out the translational GUS-GFP fusion and BaR plant selection marker gene. The AtRD29A (AtRD29) promoter gene sequence [65] was cloned into the same plasmid as a positive control of droughtinducible promoters.

\section{Transgenic $A$. thaliana plants}

The recombinant plasmids were introduced into Agrobacterium tumefaciens GV3101 strain, which was then transferred to $A$. thaliana ecotype Columbia (Col-0) by floral dip method [66]. Transgenic plants with a T-DNA insertion were identified by glufosinate-ammonium selection and confirmed by PCR. Three homozygous independent lines were obtained for each construction and $\mathrm{T}_{2}$ plants expressing GUS-GFP used in abiotic stress treatments and promoter characterization.

\section{Drought, PEG and ABA treatment of $A$. thaliana transgenic lineages}

The $A$. thaliana seeds were germinated in the soil and grown under growth chamber-controlled conditions (12 $\mathrm{h}$ photoperiod, $21^{\circ} \mathrm{C}$ temperature and $70 \%$ relative humidity). After 10 days, the seedlings were sprayed three times at intervals of 5 days with glufosinate-ammonium $(100 \mathrm{mg} / \mathrm{L})$ for positive transgenic plant selection. Four weeks old transgenic plants were carefully removed of soil moisture, and their roots were immersed in Hoagland hydroponic solution supplemented with $5 \%(\mathrm{w} / \mathrm{v})$, PEG (MW 8.000) and $50 \mu \mathrm{M}$ ABA solution to simulate drought conditions. For the drought treatment, the plants were removed from the hydroponic solution and placed on open plates. The non-stressed controls consisted of plants that were kept in Hoagland hydroponic solution. Two leaves of three plants for each full-length or modular promoter were collected after $12 \mathrm{~h}$ of treatment and immediately frozen in liquid nitrogen and stored at $-80^{\circ} \mathrm{C}$ for further extraction of RNA.

\section{Histochemical GUS assays}

To detect GUS activity in transgenic $A$. thaliana lineages, fresh leaves were incubated for $12-16 \mathrm{~h}$ at $37^{\circ} \mathrm{C}$ in 5 bromo-4-chloro-3-indolyl- $\beta$-D-glucuronic acid (X-Gluc) solution [67]. After X-Gluc incubation, the leaves were washed with water, and the chlorophyll was removed with ethanol $(70 \% \mathrm{v} / \mathrm{v})$ for approximately $10 \mathrm{~h}$. The leaves were washed and then observed under Leica Wild Heerbrugg M3Z Stereozoom Microscope (Leica, Wetzlar, Germany). For each construct, leaves were collected from at least three different transgenic plants lineages.

\section{Fluorimetric GUS assay}

The A. thaliana transgenic plants were grouped into three-plant pools and subjected to stress treatments 
(PEG, ABA, and drought) as previously described. For plants pool protein extraction, extraction buffer with $100 \mathrm{mM} \mathrm{NaH} \mathrm{PO}_{4}, 0.01 \%$ SDS, $10 \mathrm{mM}$ EDTA, 0.1\% sodium lauryl sarcosine, $0.1 \%$ Triton $\mathrm{X}-100$ and $1 \mathrm{mM}$ DTT was used. Protein extraction was performed with frozen tissue powder $(\sim 100 \mathrm{mg})$, and samples were manipulated on ice. The total soluble proteins were quantified by the Bradford method [68] and used for a fluorimetric assay. The fluorimetric GUS assay was performed in a $500 \mu \mathrm{L}$ reaction consisting of $400 \mu \mathrm{L}$ of protein extract and $100 \mu \mathrm{L}$ of $10 \mathrm{mM}$ 4-methylumbelliferyl $\beta$-D-glucuronide (MUG; Sigma, USA). The reaction was incubated for $1 \mathrm{~h}$ at $37^{\circ} \mathrm{C}$. At the start point, a $50 \mu \mathrm{L}$ reaction aliquot was removed and added to $450 \mu \mathrm{L}$ of 0.2 $\mathrm{M} \mathrm{Na}_{2} \mathrm{CO}_{3}$ stop buffer. The fluorescence of 4methylumbelliferone (4-MU) was monitored using a Versa Fluor Fluorometer (BioRad) with excitation at 365 $\mathrm{nm}$ and emission at $455 \mathrm{~nm}$. Each sample was analyzed in triplicate, and values were calculated according to a reference range of MU. GUS activity was expressed in nanomoles of MU produced per minute per microgram of soluble protein.

All GUS fluorimetric assays were repeated at least three times. The results were expressed as the mean of independent experiments with the respective standard error. Different lowercase letters above the bars indicate significant differences at $p<0.05$.

\section{GUS gene expression analysis}

The GUS gene expression was analyzed in transgenic Arabidopsis plants expressing pGmRD26::GUS, pGmRD26A:: GUS, pGmRD26B::GUS and pAtRD29::GUS. The gene expression level was monitored by qRT-PCR using three biological and two technical replicates, as previously described for soybean genes; the expression levels were normalized using ACT2 (AT3G18780 [69]) and GAPDH (AT1G13340 [70]) as endogenous controls. The endogenous gene stability was determined by G-norm algorithm, from Q-base package, and the $\mathrm{M}$-value is 0.86 and 0.79 for $A C T 2$ and GAPDH, respectively. The primers used are described in Additional file 3: Table S2.

\section{Supplementary information}

Supplementary information accompanies this paper at https://doi.org/10. 1186/s12896-019-0561-3.

\footnotetext{
Additional file 1: Figure S1. GmNAC085 expression profile in soybean (Williams 82) under multiple stresses. To determine the gene expression profile of the GmNAC085 gene, the soybean seedlings were submitted to different stress conditions (ABA, PEG, AS, Tun, and drought), and the gene expression were analyzed in leaves and roots by GRT-PCR. The fold change values were calculated in comparison of plants treated with untreated plants ( $0 \mathrm{~h}$ ). CYP2 and ELF1A were used as endogenous controls for normalization. The relative gene expression was calculated by the ${ }_{2-}{ }^{\Delta \Delta \mathrm{Ct}}$ method in biological triplicates $(n=3)$. The bars represent standard
}

errors and the asterisks (*) indicate statistical significance determined by the Student's t-test $(P \leq 0.05)$.

Additional file 2: Table S1. Cis-regulatory elements related to drought revealed in the $\mathrm{pGmRD26}$ soybean promoter and the A. thaliana promoter RD29 ( $p$-value of 0.05).

Additional file 3: Table S2. Primer sequences used in the GRT-PCR analysis.

\section{Abbreviations}

ABA: Abscisic acid; ABRE: ABA-responsive element; AtRD29: Arabidopsis thaliana responsive to desiccation 29; DREB: Dehydration-responsive element binding; MYB: Myeloblastosis; MYC: Myelocytomatosis; NAC: Nitrogen assimilation control; PEG: Polyethylene glycol; pGmRD26: Glycine max responsive to desiccation 26 promoter; TFs: Transcription factors

\section{Acknowledgments}

Not Applicable.

\section{Authors' contributions}

EOF and BPM executed the experiments and wrote the first draft of the manuscript; ITLT and FBMA executed GPCR and fluorimetric assay; RMA and MELS performed abiotic stress experiments on soybean; JAC and AGBL transformed $A$. thaliana with $\mathrm{pGmRD26}$ full-length promoter and modular promoters; MF and CVM performed statistical analysis, MAF, EPBF and MFGS designed the study, revised and finalized the manuscript. All contributing authors have read and approved the final manuscript.

\section{Funding}

This work was supported by EMBRAPA, CNPq (INCT 465480/2014-4), CAPES and FAPDF (INCT 0193-001.265/2017). CAPES provides fellowship grants to EOF, BPM, FBMA and JAC. CNPq provides a fellowship grant to AGBL. The funding body did not participate of the study design, data collection and analysis and manuscript writing.

\section{Availability of data and materials}

The datasets supporting the conclusions of this article are included within the article and its additional files. All plant materials were obtained from Embrapa Genetic Resources and Biotechnology. Brasília- Brazil.

Ethics approval and consent to participate

Not applicable.

Consent for publication

Not applicable.

\section{Competing interests}

The authors declare that they have no competing interests.

\section{Author details}

${ }^{1}$ Embrapa Genetic Resources and Biotechnology, Brasília, DF, Brazil. ${ }^{2}$ Federal University of Brasília, Brasília, DF, Brazil. ${ }^{3}$ Federal University of Viçosa, Viçosa, MG, Brazil. ${ }^{4}$ Federal University of Rio Grande do Sul, Porto Alegre, RS, Brazil. ${ }^{5}$ Agricultural Research Company of Minas Gerais State, Uberaba, MG, Brazil. ${ }^{6}$ Catholic University of Brasilia - Post-Graduation Program in Genomic Sciences and Biotechnology, Brasília, DF, Brazil. ${ }^{7}$ National University of Medical Sciences, Rawalpindi, Punjab, Pakistan. ${ }^{8}$ Federal University of Rio de Janeiro, Rio Janeiro, RJ, Brazil. 'Embrapa Semi-Arid, Petrolina, PE, Brazil.

Received: 31 May 2019 Accepted: 13 September 2019 Published online: 20 November 2019

\section{References}

1. Sheffield J, Wood EF, Roderick ML. Little change in global drought over the past 60 years. Nature. 2012;491:435-8.

2. Leng G, Hall J. Crop yield sensitivity of global major agricultural countries to droughts and the projected changes in the future. Sci Total Environ. 2019; 654:811-21.

3. Tuteja N. Cold, salinity and drought stress. Plant Stress Biol. 2009;149:137-59. 
4. Fang Y, Xiong L. General mechanisms of drought response and their application in drought resistance improvement in plants. Cell Mol Life Sci. 2015;72:673-89

5. Nakashima K, Yamaguchi-Shinozaki K. ABA signaling in stress-response and seed development. Plant Cell. 2013;32:959-70.

6. Takahashi F, Takehiro S, Yuriko O, Shigeyuki B, Yuki K, Naoshi D, et al. A small peptide modulates stomatal control via abscisic acid in long-distance signalling. Nature. 2018:556:235-8.

7. Abe H, Yamaguchi-Shinozaki K, Urao T, Iwasaki T, Hosokawa D, Shinozaki K Role of MYC and MYB homologs in drought- and abscisic acid-regulated gene expression. Plant Cell. 1997;9:1859-68.

8. Zhao L, Hu Y, Chong K, Wang T. ARAG1, an ABA-responsive DREB gene, plays a role in seed germination and drought tolerance of rice. Ann Bot. 2010;105:401-9.

9. Yoshida T, Fujita Y, Sayama H, Kidokoro S, Maruyama K, Mizoi JK, Shinozaki Y. AREB1, AREB2, and ABF3 are master transcription factors that cooperatively regulate $A B R E-d e p e n d e n t A B A$ signaling involved in drought stress tolerance and require ABA for full activation. Plant J. 2010;61:672-85

10. Li J, Xu Y, Niu Q, He L, Teng Y, Bai S. Abscisic acid (ABA) promotes the induction and maintenance of pear (Pyrus pyrifolia white pear group) flower bud endodormancy. Int J Mol Sci. 2018;19:1-15.

11. Nguyen KH, Mostofa MG, Watanabe Y, Tran CD, Rahman MM, Tran LSP. Overexpression of GmNAC085 enhances drought tolerance in Arabidospis by regulating glutathione biosynthesis, redox balance and glutathione dependent detoxification of reactive oxygen species and methylglyoxal. Environ Exp Botany. 2018;161:242-54.

12. Mizoi J, Shinozaki K, Yamaguchi-Shinozaki K. AP2/ERF family transcription factors in plant abiotic stress responses. Biochim Biophys Acta. 1819;2012:86-96.

13. Kasuga M, Miura S, Shinozaki K, Yamaguchi-Shinozaki K. A combination of the Arabidopsis DREB1A gene and stress-inducible rd29A promoter improved drought- and low-temperature stress tolerance in tobacco by gene transfer. Plant Cell Physiol. 2004;45:346-50.

14. Behnam B, Kikuchi A, Celebi-Toprak F, Yamanaka S, Kasuga M, YamaguchiShinozaki K, Watanabe KN. The Arabidopsis DREB1A gene driven by the stress-inducible rd29A promoter increases salt-stress tolerance in proportion to its copy number in tetrasomic tetraploid potato (Solanum tuberosum). Plant Biotechnol. 2006;23:169-77.

15. Engels C, Fuganti-Pagliarini R, Marin SRR, Marcelino-Guimarães FC, Oliveira MCN, Kanamori N, et al. Introduction of the rd29A: AtDREB2A CA gene into soybean (Glycine max L. Merril) and its molecular characterization in leaves and roots during dehydration. Genet Mol Biol. 2013;36:556-65.

16. Kasuga M, Liu Q, Miura S, Yamaguchi-Shinozaki K, Shinozaki K. Improving plant drought, salt and freezing tolerance by gene transfer of a single stress-inducible transcription factor. Nat Biotechnol. 1999;17:287-91.

17. Bihmidine S, Lin J, Stone JM, Awada T, Specht JE, Clemente TE. Activity of the Arabidopsis RD29A and RD29B promoter elements in soybean under water stress. Planta. 2013;237:55-64.

18. Kidokoro S, Watanabe K, Ohori T, Moriwaki T, Maruyama K, Mizoi J. Soybean DREB 1/CBF type transcription factors function in heat and drought as well as cold stress responsive gene expression. Plant J. 2015;81:505-18.

19. Sarker S, Biswas S, Shahed A, Razzaque S, Seraj Zl. Cloning, characterization and analysis of the Arabidopsis RD29A promoter for its inducible expression in rice under salinity and drought stress. Biores Commun. 2016;2(1):139-45.

20. Nobres P, Patreze CM, Waltenberg FP, Marcelo FC, Tavano EC, Mendes BMJ, et al. Characterization of the promoter of the homeobox gene $\mathrm{CaHB12}$ in Coffea arabica. Trop Plant Biol. 2016;9(1):50-62.

21. Melo BP, Fraga OT, Silva JCF, Ferreira DO, Brustolini OJB, Carpinetti PA Machado JPB, Reis PAB, Fontes EPB. Revisitinh the soybean GmNAC superfamily. Front Plant Sci. 2018;9:1864.

22. Rodrigues FA, Marcolino J, Carvalho JDFC, Nascimento LCD, Neumaier N, Farias JRB, et al. Subtractive libraries to prospect differentially expressed genes in soybean plants submitted to water deficit. Genet Mol Biol. 2012;35: 304-14

23. Takasaki H, Maruyama K, Takahashi F, Fujita M, Yoshida T, Nakashima K, et al. SNAC-as, stress-responsive NAC transcription factors, mediate ABA-inducible leaf senescence. Plant J. 2015:84:1114-23.

24. Li S, Gao J, Yao L, Ren L, Zhu X, Gao S, et al. The role of ANAC072 in the regulation of chlorophyll degradation during age- and dark-induced leaf senescence. Plant Cell Rep. 2016;35:1729.

25. Simpson SD, Nakashima K, Narusaka Y, Seki M, Shinozaki K, YamaguchiShinozaki K. Two different novel cis-acting elements of e1, a clpA homologous Arabidopis gene function in induction by dehydration stress and dark-induced senescence. Plant J. 2003:33:259-70.

26. Hartmann U, Sagasser M, Mehrtens F, Stracke R, Weisshaar B. Differential combinatorial interactions of cis-acting elements recognized by R2R3-MYB, $B Z I P$, and BHLH factors control light-responsive and tissue-specific activation of phenylpropanoid biosynthesis genes. Plant Mol Biol. 2005;57:155-71.

27. Agarwal M, Hao Y, Kapoor A, Dong CH, Fujii H, Zheng X, et al. A R2R3 type MYB transcription factor is involved in the cold regulation of CBF genes and in acquired freezing tolerance. J Biol Chem. 2006;281:37636-45.

28. Hattori T, Totsuka M, Hobo T, Kagaya Y, Yamamoto TA. Experimentally determined sequence requirement of ACGT-containing abscisic acid response element. Plant Cell Physiol. 2002;43:136-40.

29. Kizis D, Pages M. Maize DRE-binding proteins DBF1 and DBF2 are involved in rab17 regulation through the drought-responsive element in an ABAdependent pathway. Plant J. 2002;30:679-89.

30. Abe H, Urao T, Ito T, Seki M, Shinozaki K, Yamaguchi-Shinozaki K. Arabidopsis AtMYC2 (bHLH) and AtMYB2 (MYB) function as transcriptional activators in abscisic acid signaling. Plant Cell. 2003;15:63-78.

31. Suzuki M, Ketterling MG, McCarty DR. Quantitative statistical analysis of cis regulatory sequences in ABANP1 and CBF/DREB1 regulated genes of Arabidopsis. Plant Physiol. 2005;139:437-47.

32. Kang JY, Choi HI, Im MY, Kim SY. Arabidospis basic leucine zipper proteins that mediate stress-responsive abscisic acid signaling. Plant Cell. 2002;14 343-57.

33. Choi HI, Park HJ, Park JH, Kim S, Im MY, Seo HH, et al. Arabidopsis calciumdependent protein kinase AtCPK32 interacts with ABF4, a transcriptional regulator of abscisic acid-responsive gene expression and modulates its activity. Plant Physiol. 2005;139:1750-61.

34. Urao T, Yamaguchi-Shinozaki K, Urao S, Shinozaki K. An Arabidopsis myb homolog is induced by dehydration stress and its gene product binds to the conserved MYB recognition sequence. Plant Cell. 1993;5:1529-39.

35. Solano R, Nieto C, Avila J, Canas L, Diaz I, Paz-Ares J. Dual DNA binding specificity of a petal epidermis-specific MYB transcription factor (MYB.Ph3) from Petunia hybrida. EMBO J. 1995:14:1773-84.

36. Yang T, Poovaiah BW. Arabidopsis calmodulin-binding/CGCG box DNAbinding protein family involved in multiple signaling pathways in plants. J Biol Chem. 2002;277:45049-58.

37. Stalberg K, Ellerstom M, Ezcurra I, Ablov S, Rask L. Disruption of an overlapping E-box/ABRE motif abolished high transcription of the napA storage-protein promoter in transgenic Brassica napus seeds. Planta. 1996; 199:515-9.

38. Kim SY, Chung HJ, Thomas TL. Isolation of a novel class of bZIP transcription factors that interact with ABA-responsive and embryospecification elements in the Dc3 promoter using a modified yeast onehybrid system. Plant J. 1997;11:1237-51.

39. Finkelstein RR, Lynch TJ. The Arabidopsis abscisic acid response gene ABI5 encodes a basic leucine zipper transcription factor. Plant Cell. 2000:12:599_ 609.

40. Kaplan B, Davydov O, Knight H, Galon Y, Knight MR, Fluhr R, et al. Rapid transcriptome changes induced by cytosolic $\mathrm{Ca}^{2+}$ transients reveal ABRErelated sequences as $\mathrm{Ca}^{2+}$-responsive cis elements in Arabidopsis. Plant Cell. 2006;18:2733-48.

41. Fujita M, Fujita Y, Maruyama K, Seki M, Hiratsu K, Ohme-Takagi M, et al. A dehydration-induced NAC protein, RD26, is involved in a novel ABAdependent stress-signaling pathway. Plant J. 2004:39:863-76.

42. Thu NBA, Hoang XLT, Doan H, Nguyen TH, Bui D, Thao NP, Tran LS. Differential expression analysis of a subset of GmNAC genes in shoots of two contrasting drought-responsive soybean cultivars DT51 and MTD720 under normal and drought conditions. Mol Biol Rep. 2014;41:5563.

43. Shinozaki K, Yamaguchi-Shinozaki K. Gene networks involved in drought stress response and tolerance. J Exp Bot. 2007;58:221-7.

44. Tran LS, Quach TN, Guttikonda SK, Aldrich DL, Kumar R, Neelakandan A et al. Molecular characterization of stress-inducible GmNAC genes in soybean. Mol Gen Genomics. 2010;281:647-64.

45. Lu PL, Chen NZ, An R, Su Z, Qi BS, Ren F, et al. A novel drought-inducible gene, ATAF1, encodes a NAC family protein that negatively regulates the expression of stress-responsive genes in Arabidopsis. Plant Mol Biol. 2007;63: 289-305.

46. Jensen MK, Lindemose S, Mais F, Reimer JJ, Nielsen M, Perera V, et al. ATAF1 transcription factor directly regulates abscisic acid biosynthetic gene NCED3 in A. thaliana. FEBS Open Bio. 2013;29:321-7. 
47. Ye Y, Ding Y, Jiang $Q$, Wang F, Sun J, Zhu C. The role of receptor-like protein kinases (RLKs) in abiotic stress response in plants. Plant Cell Rep. 2017;36:235-42.

48. Zhu J-K. Salt and drought stress signal transduction in plants. Rev Plant Biol. 2002:53:247-73.

49. Nakashima K, Takasaki H, Mizoi J, Shinozaki K, Yamaguchi-Shinozaki K. NAC transcription factors in plant abiotic stress responses. Biochim Biophys Acta. 1819:2012:97-103.

50. Guiltinan M, Marcotte W, Quatrano R. A plant leucine zipper protein that recognizes an abscisic acid response element. Science. 1990;250:267-71.

51. Mehrotra R, Sethi S, Zutshi I, Bhalothia P, Mehrotra S. Patterns and evolution of ACGT repeat cis-element landscape across four plant genomes. BMC Genomics. 2013;14:2-11.

52. Nakashima K, Tran LS, Van Nguyen D, Fujita M, Maruyama K, Todaka D. Functional analysis of a NAC-type transcription factor OsNAC6 involved in abiotic and biotic stress-responsive gene expression in rice. Plant J. 2006;51: 617-30.

53. Yamaguchi-Shinozaki K, Shinozaki K. Organization of cis-acting regulatory elements in osmotic- and cold-stress-responsive promoters. Trends Plant Sci. 2005;10:88-94.

54. Bhargava S, Sawant K. Drought stress adaptation: metabolic adjustment and regulation of gene expression. Plant Breed. 2013;132:21-32.

55. Hernandez-Garcia CM, John JJ. Identification and validation of promoters and cis-acting regulatory elements. Plant Sci. 2014;217:109-19.

56. Martinez MC, Goossens A. Combinatorial transcriptional control of plant specialized metabolism. Trends Plant Sci. 2018:23:324-36.

57. Conforte AJ, Guimarães-Dias F, Neves BAC, Bencke-Malato M, Whipps DF, Alves-Ferreira M. Isolation and characterization of a promoter responsive to salt, osmotic and dehydration stresses in soybean. Gen Mol Biol. 2017;40: 226-37.

58. Behnam B, luchi S, Fujita M, Fujita Y, Takasaki H, Osakabe Y, et al. Characterization of the promoter region of an Arabidopsis gene for 9-cisepoxycarotenoid dioxygenase involved in dehydration-inducible transcription. DNA Res. 2013;20:315-24.

59. Imtiaz M, Yang Y, Liu R, Xu Y, Khan MA, Wei Q, Gao J, Hong B. Identification and functional characterization of the BBX24 promoter and gene from Chrysanthemum in a. Plant Mol Biol. 2015:89:1-19.

60. Goodstein DM, Shu S, Howson R, Neupane R, Hayes RD, Fazo J, et al. Phytozome: a comparative platform for green plant genomics. Nucleic Acids Res. 2012;40:1178-86.

61. Tamura K, Dudley J, Nei MS. MEGA4: molecular evolutionary genetics analysis (MEGA) software ver. 4.0. Mol Biol Evol. 2007;24:1596-9.

62. Jian B, Liu B, Bi Y, Hou W, Wu C, Han T. Validation of internal control for gene expression study in soybean by quantitative real-time PCR. BMC Mol Biol. 2008;9:1-14

63. Cartharius K, Frech K, Grote K, Klocke B, Haltmeier M, Klingenhoff A, et al, MatInspector and beyond: promoter analysis based on transcription factor binding sites. Bioinformatics. 2005:21:2933-42.

64. Ho Sui SJ, Mortimer JR, Arenillas DJ, Brumm J, Walsh CJ, Kennedy BP, Wasserman WW. OPOSSUM: identification of over-represented transcription factor binding sites in co-expressed genes. Nucleic Acids Res. 2005;33:3154-64.

65. Yamaquchi-Shinozaki K, Shinozaki K. Characterization of the expression of a desiccation-responsive rd29 gene of $A$. thaliana and analysis of its promoter in transgenic plants. Mol Gen Genet. 1993;236:331-40.

66. Clough SJ, Bent AF. Floral dip: a simplified method for agrobacteriummediated transformation of A. thaliana. Plant J. 1998;16:735-43.

67. Jefferson RA. The GUS reporter gene system. Nature. 1989:342:837-8.

68. Bradford MM. A rapid and sensitive method for the quantitation of microgram quantities of protein utilizing the principle of protein-dye binding. Anal Biochem. 1976;72:248.

69. An YQ, McDowell JM, Huang S, McKinney EC, Chambliss S, Meagher RB. Strong constitutive expression of the Arabidopsis ACT2/ACT8 actin subclass in vegetative tissues. Plant J. 1996;10:107-21.

70. Bustin SA. Absolute quantification of mRNA using real-time reverse transcription polymerase chain reaction assays. J Mol Endocrinol. 2000;25:169-93.

\section{Publisher's Note}

Springer Nature remains neutral with regard to jurisdictional claims in published maps and institutional affiliations.

Ready to submit your research? Choose BMC and benefit from:

- fast, convenient online submission

- thorough peer review by experienced researchers in your field

- rapid publication on acceptance

- support for research data, including large and complex data types

- gold Open Access which fosters wider collaboration and increased citations

- maximum visibility for your research: over $100 \mathrm{M}$ website views per year

At $\mathrm{BMC}$, research is always in progress.

Learn more biomedcentral.com/submissions 